\title{
BMJ Open Is manager support related to workplace productivity for people with depression: a secondary analysis of a cross-sectional survey from 15 countries
}

\author{
Sara Evans-Lacko, Martin Knapp
}

To cite: Evans-Lacko S, Knapp M. Is manager support related to workplace productivity for people with depression: a secondary analysis of a cross-sectional survey from 15 countries. BMJ Open 2018;8:e021795. doi:10.1136/ bmjopen-2018-021795

- Prepublication history for this paper is available online. To view these files, please visit the journal online (http://dx.doi. org/10.1136/bmjopen-2018021795).

Received 25 January 2018

Revised 7 April 2018

Accepted 10 April 2018

\section{Check for updates}

Personal Social Services,

Research Unit, London School of Economics and Political Science, London, UK

Correspondence to

Dr Sara Evans-Lacko; s.evans-lacko@Ise.ac.uk

\section{ABSTRACT}

Objectives To examine variations in manager reactions and support for people with depression and to investigate how these reactions are related to (1) absenteeism and (2) presenteeism due to depression among employees with self-reported depression across 15 diverse countries. Design Secondary data analysis of cross-sectional survey data.

Setting 15 countries, diverse in geographical region and gross domestic product (GDP): Brazil, Canada, China, Denmark, France, Germany, Great Britain, Italy, Japan, Mexico, Spain, South Africa, South Korea, Turkey and the USA.

Participants 16018 employees and managers (approximately 1000 per country).

Primary and secondary outcome measures We assessed level of absenteeism as measured by number of days taken off work because of depression and presenteeism score.

Results On average, living in a country with a greater prevalence of managers saying that they avoided talking to the employee about depression was associated with employees with depression taking more days off work (B 4.13, 95\% $\mathrm{Cl} 1.68$ to 6.57). On average, living in a country with a higher GDP was marginally associated with employees with depression taking more days off of work $(p=0.09)$. On average, living in a country with a greater prevalence of managers actively offering help to employees with depression was associated with higher levels of presenteeism (B 7.08, 95\% $\mathrm{Cl} 6.59$ to 7.58). Higher country GDP was associated with greater presenteeism among employees with depression (B 3.09, $95 \% \mathrm{Cl} 2.31$ to 3.88 ).

Conclusions Manager reactions were at least as important as country financial resources. When controlling for country GDP, working in an environment where managers felt comfortable to offer help and support to the employee rather than avoid them was independently associated with less absenteeism and more presenteeism.

\section{BACKGROUND}

Although depression is experienced by a large proportion of employees, it is often seen as a taboo issue. More than $70 \%$ of people with mental illness actively conceal their mental illness from others, and most

\section{Strengths and limitations of this study}

- Our data come from a unique dataset including both employee and manager experiences of depression from 15 diverse countries.

- Data were cross-sectional, so it was not possible to examine pathways or mechanisms to increased productivity.

- Depression diagnosis was determined via self-report and did not include severity or type of symptoms, though distribution of respondent characteristics with depression are similar to findings from other epidemiological studies.

- Response rates were relatively low, though our samples were geographically representative and we used quota sampling to ensure equal distributions of age and gender.

- We did not have information on mental health policies or employment assistance programmes available in the workplace.

of those who conceal do so because they fear discrimination when looking for or keeping a job. ${ }^{12}$ We know that supportive managers and workplace practices are associated with greater openness and disclosure, in addition to more positive attitudes towards employees with depression. ${ }^{3}$ However, many individuals with depression face discrimination in the workplace. ${ }^{24}$ As a result, individuals with mental health problems such as depression often avoid disclosing their problem at work or seeking help because they fear negative employer and coworker reactions, as well as repercussions for their career. ${ }^{5}{ }^{6}$ Workplace disability policy covers mental illness in many countries; however, people with mental illness face significant disadvantage in finding and keeping work, in part because employers often lack training and understanding of mental health issues. ${ }^{78}$ Indeed, relative to other disabilities, mental illness is associated with the greatest disadvantage in terms of employment rates. ${ }^{49}$ All of these issues 
contribute to low levels of disclosure and help-seeking for depression among employees.

Given the high economic costs of depression to employers, ${ }^{1011}$ workplace interventions have been developed to support individuals with depression. There is also preliminary evidence that general health promotion programmes can improve productivity in the workplace. ${ }^{12}$ Yet there are additional barriers for mental health-related programmes as many individuals choose not to seek help for mental health problems in the workplace ${ }^{6}$ due to underlying issues of stigma and discrimination. Thus, before implementing programmes for prevention and treatment of mental illness in the workplace, it may be important to address underlying issues related to stigma and support. Indeed, these could represent key factors which allow individuals to return to or remain in work and to be productive in their roles.

Consequently, we investigate the relationship between manager reactions and support for people with depression and workplace productivity across 15 countries, diverse in geographical region and GDP: Brazil, Canada, China, Denmark, France, Germany, Great Britain, Italy, Japan, Mexico, Spain, South Africa, South Korea, Turkey and the USA. First, we examined variation in active strategies to support an employee with depression rather than an approach which avoids or ignores the issue by calculating country prevalence of managers reporting: (1) offering help to employees with depression and (2) avoiding talking about depression with the employee. Second, we considered how these reactions alongside individual employee characteristics related to (1) absenteeism and (2) presenteeism among employees with depression.

\section{METHODS}

\section{Data source}

We performed secondary data analysis on the Global IDEA (Impact of Depression in the Workplace in Europe Audit) survey data which collected data from employed persons across 15 diverse countries, 7 in Europe: Denmark, France, Germany, Italy, Spain, UK, Turkey—and 8 others-Brazil, Canada, China, Japan, South Korea, Mexico, South Africa and the USA. Participants were recruited through an online market research panel. Before joining the panel, participants went through a screening process to validate their personal data which included: removal of duplicates, validation of name and surname through name/gender match or mismatch/misspelling as compared with library of names, country validation based on internet protocol address used to identify unique users, validation of town and zip/postal code according to official lists, checking for valid correlations between sociodemographic data (gender, age of parents and children) and validation of contact information. Individuals who worked in advertising and/or market research, and those aged under 16 years were excluded.
Employed people across the selected countries were sampled from the online research panels. Selected panel members were invited via email to participate in the survey by Ipsos MORI (www.ipsos-mori.com/). Quotas were set to include equal distributions of age and gender, and the sample was designed to be geographically representative of each country. In addition, as managers were considered of key interest, $10 \%$ of the sample for each country was represented by managers. Response rates varied by country and ranged from around $5 \%$ in China to $39 \%$ in France. Questionnaires were collected from approximately 1000 respondents per country.

\section{Measures}

Sociodemographic information included gender, age band (16-24, 25-44 and 45-64 years), gender, education level completed (tertiles were created for each country to indicate locally relevant high, medium and low education categories). Individuals were also asked to describe whether the company in which they were employed was small (1-50 employees), medium (51-250 employees) or large (more than 250 employees).

Previous diagnosis of depression was determined via self-report by asking respondents: Have you ever personally been diagnosed as having depression by a doctor/ medical professional?

\section{Country variables}

We used data from the IDEA survey to describe the overall population prevalence of employees with a diagnosis of depression. Managers who said that they had one or more employees with depression in the past were asked how they responded to the employee. We calculated national prevalence of those who reported they: (1) avoided talking to them about it and (2) discussed with them and asked if there was anything (the manager) could do to help. Figures for GDP per capita (US\$) for each participating country were taken from the World Bank. ${ }^{13}$

\section{Work performance}

Absenteeism was assessed using the following question: 'The last time you experienced depression, how many working days did you have to take off work because of your depression'. Absenteeism data were available in all 15 countries. Presenteeism was assessed using WHO Health and Work Performance Questionnaire. ${ }^{14} 15$ Presenteeism data were only available in a subset of eight countries (Brazil, Canada, China, Japan, South Korea, Mexico, South Africa and the USA) in which the surveys were conducted at a slightly later date.

\section{Statistical analysis}

Individual participant (gender, age, education and income) and aggregated country characteristics were described overall and for individuals with and without depression. There were no missing data on variables of interest. Two generalised linear models were used to examine the multivariable factors associated with: (1) greater absenteeism as measured by number of days taken 
off work because of depression (2) a higher presenteeism score. Country contextual characteristics were computed as an average rating for each country across respondents, and each variable was standardised (ie, z-score was computed). Poststratification weights, based on gender, age and region, which were aligned with nationally representative figures, were used in all analyses.

We used generalised estimating equations (GEEs) with robust variance estimates to model within-country correlations. ${ }^{16}$ We selected GEE instead of mixed-regression models as we were interested in understanding the influence of overall cultural factors rather than individual country-level effects. Thus, a population average model was more appropriate for our research question. As GEE is a non-likelihood-based method, Pan's QIC (quasi-likelihood under the independence model criterion) was used for variable selection and selecting the working correlation matrix. QIC is a statistic which generalises Akaike information criterion to GEE models by replacing likelihood estimation with quasi-likelihood estimation and making adjustments for the penalty term. A lower QIC value indicates better model fit. ${ }^{17}$ All analyses were carried out using SAS V.9.3 and Stata V.11.

This study was classified as exempt by the King's College London, Psychiatry, Nursing and Midwifery Research Ethics Subcommittee. Data collection was performed independently by Ipsos MORI in accordance with the standards of European Society for Opinion and Market Research (ESOMAR), Alliance of International Market Research Institutes (AIMRI) and European Federation of Market Research Associations (EFAMRO) in Europe, and is in line with the data protection act 1998 .

\section{Patient and public involvement}

There was no patient and public involvement in the development of the research question or the selection of outcome measures. All analyses were performed on secondary data.

\section{RESULTS}

Individual sociodemographic and employment characteristics are described in table 1 for individuals with and without a reported history of depression.

As expected, given the diversity of countries-and hence diversity of workplace cultures, policies and economic and employment contexts-there was wide variation in responses by managers to responses to employees with depression and reported training and/ or support for managers across the 15 countries. In general, managers in Asian countries tended to avoid employees with depression rather than use active support strategies. Managers in China and South Korea also reported low levels of support in dealing with depression in the workplace and were less likely to offer active support (see figures 1 and 2).

\section{Factors associated with greater absenteeism}

Table 2 describes the individual and country contextual characteristics associated with greater absenteeism among employees with depression. In terms of individual characteristics, individuals with high levels of education relative to those with low levels of education took more days off work because of their depression. Those working in larger companies relative to smaller companies took fewer days off work. In terms of country contextual characteristics, on average, living in a country with a greater prevalence of managers saying that they avoided talking to the employee about depression was associated with employees with depression taking more days off of work. On average, living in a country with a higher GDP was marginally associated with employees with depression taking more days off work $(\mathrm{p}=0.09)$.

\section{Factors associated with greater presenteeism}

Table 3 describes the individual and country contextual characteristics associated with greater presenteeism among employees with depression. In terms of individual characteristics, employees with depression who were male, in the older age group (ages 45-64 relative to 16-24) and in the medium relative to low education level tended to have higher levels of presenteeism. Employees with depression who were in the middle age group (25-44 relative to 16-24) tended to have lower levels of presenteeism. In terms of country contextual characteristics, on average, living in a country with a greater prevalence of managers actively offering help to employees with depression had higher levels of presenteeism. Higher country GDP was associated with greater presenteeism among employees with depression.

\section{DISCUSSION}

Substantial research demonstrates that depression is experienced by a large proportion of the workforce and associated with high costs to employers. ${ }^{11}{ }^{18}$ Our findings add to this literature and suggest that manager reactions to employees with depression can reflect broad cultural and organisational features that directly relate to employee productivity. This strengthens the economic case for supporting the development and implementation of effective policies and practices for managers to be able to actively support an employee with depression. Additionally, certain personal characteristics may make individuals prone to take more days off work (absenteeism) and/or reduce workplace performance (presenteeism). This suggests that additional support might be provided to more vulnerable subgroups to address this difference in experience.

Workplace policies and practices in relation to mental illness vary widely across countries and organisations..$^{19} 20$ Our findings show that there is substantial variation across countries in terms of how managers were able to actively support rather than avoid employees with depression in the workplace, and likely also variation in terms of how 
Table 1 Characteristics of employee respondents, overall and by history of depression (weighted per cent, 95\% Cl)

\begin{tabular}{|c|c|c|c|}
\hline Respondent characteristics & Overall sample $n=16018$ & $\begin{array}{l}\text { Individuals reporting a } \\
\text { history of depression } \\
n=2985\end{array}$ & $\begin{array}{l}\text { Individuals with no } \\
\text { history of depression } \\
n=13033\end{array}$ \\
\hline \multicolumn{4}{|l|}{ Gender } \\
\hline Male & 55.2 (54.4 to 56.0$)$ & 43.3 (41.4 to 45.1$)$ & 58.0 (57.1 to 58.9$)$ \\
\hline Female & 44.8 (44.0 to 45.6$)$ & 56.7 (54.9 to 58.6$)$ & 42.0 (41.1 to 42.9$)$ \\
\hline \multicolumn{4}{|l|}{ Age } \\
\hline $16-24$ & 34.7 (33.9 to 35.5$)$ & 32.8 (31.0 to 34.6$)$ & 35.2 (34.3 to 36.1$)$ \\
\hline $25-44$ & 51.4 (50.6 to 52.2$)$ & $53.2(51.3$ to 55.1$)$ & $51.0(50.1$ to 51.9$)$ \\
\hline $45-64$ & 13.9 (13.3 to 14.5$)$ & $14.0(12.7$ to 15.4$)$ & 13.9 (13.2 to 14.5$)$ \\
\hline \multicolumn{4}{|l|}{ Education } \\
\hline Low & 42.5 (41.2 to 43.8$)$ & 41.9 (39.0 to 44.9$)$ & 42.6 (41.1 to 44.2$)$ \\
\hline Medium & 22.3 (21.2 to 23.4$)$ & 23.5 (21.1 to 26.0$)$ & 22.0 (20.8 to 23.2) \\
\hline High & 35.2 (33.9 to 36.5$)$ & 34.5 (31.6 to 37.4$)$ & 35.3 (33.9 to 36.8 ) \\
\hline \multicolumn{4}{|l|}{ Working status } \\
\hline Full time & 72.6 (71.9 to 73.4$)$ & 66.4 (64.6 to 68.2$)$ & 74.1 (73.3 to 74.9$)$ \\
\hline Part time & 22.3 (21.6 to 23.0$)$ & $27.3(25.7$ to 29.0$)$ & 21.1 (20.4 to 21.9$)$ \\
\hline Previously employed in last 12 months & $5.1(4.7$ to 5.4$)$ & $13.7(5.3$ to 7.1$)$ & $4.8(4.4$ to 5.1$)$ \\
\hline \multicolumn{4}{|l|}{ Marital status } \\
\hline Single & 28.3 (27.6 to 29.1$)$ & 29.0 (26.8 to 31.3$)$ & 28.3 (27.5 to 29.1$)$ \\
\hline Married/cohabitating & $62.8(61.6$ to 64.2$)$ & 58.0 (53.8 to 62.2$)$ & $64.0(62.5$ to 65.9$)$ \\
\hline Divorced separated & 7.3 (6.8 to 7.9$)$ & $10.9(8.8$ to 13.0$)$ & 6.3 (5.7 to 6.9$)$ \\
\hline Widowed & 0.9 (0.7 to 1.0$)$ & 1.7 (1.1 to 2.3$)$ & 0.7 (0.6 to 0.9$)$ \\
\hline Refused & 0.7 (0.5 to 0.9$)$ & 0.3 (0.1 to 0.7$)$ & 0.7 (0.5 to 0.9$)$ \\
\hline \multicolumn{4}{|l|}{ Company size } \\
\hline Small (1-50 employees) & 45.1 (44.0 to 46.1$)$ & 44.1 (41.7 to 46.5$)$ & 45.3 (44.2 to 46.4$)$ \\
\hline Medium (51-250 employees) & $18.9(18.1$ to 19.7$)$ & 19.0 (17.1 to 21.0$)$ & 18.8 (17.9 to 19.7$)$ \\
\hline Large (250 + employees) & 32.2 (31.2 to 33.2) & 33.7 (31.4 to 36.1$)$ & 31.9 (30.9 to 33.0$)$ \\
\hline Do not know & 3.8 (3.4 to 4.2 ) & 3.1 (2.3 to 4.0$)$ & 4.0 (3.5 to 4.4$)$ \\
\hline
\end{tabular}

managers were supported to do so. Existing research suggests that even in high-income countries, effective workplace policies for prevention and support of mental health problems are often lacking: a 2015 OECD report noted that no countries demonstrated an advanced strategy for helping employees with mental health problems at work, though some individual companies are developing rigorous approaches. ${ }^{21}$ Few studies have reported data including low-income and middle-income countries and further research is needed to better understand the range of strategies and practices used in countries with low or moderate financial resources. ${ }^{22}$

Workplace policies and practices are likely to reflect broader sociocultural attitudes and beliefs about mental health and societal values about investment in prevention and support for people with mental health problems. ${ }^{21} 23$ There is research which highlights relatively high levels of concealment in Asian countries such as Japan and China, in comparison to other Western countries, ${ }^{24-27}$ and this may influence workplace culture in relation to openness and comfort in discussing mental health issues. Previous research has shown that a cultural context which is more open and accepting of mental illness is associated with higher rates of help-seeking, antidepressant use and empowerment. ${ }^{28} 29$

Other country contextual factors such as country GDP and financial resources can also influence the availability of support and potential for investment. Our study found that country GDP was positively related to presenteeism and marginally negatively related to absenteeism. However, our study also showed that manager reactions were at least as important as country financial resources. We found that, when controlling for country GDP, working in an environment where managers felt comfortable to offer help and support to the employee rather than avoid them was independently associated with lower absenteeism and more presenteeism. We know from other research that economic indicators such as unemployment rate and decline in GDP can be positively correlated with stigma. ${ }^{29}$ However, both stigma and manager reactions 


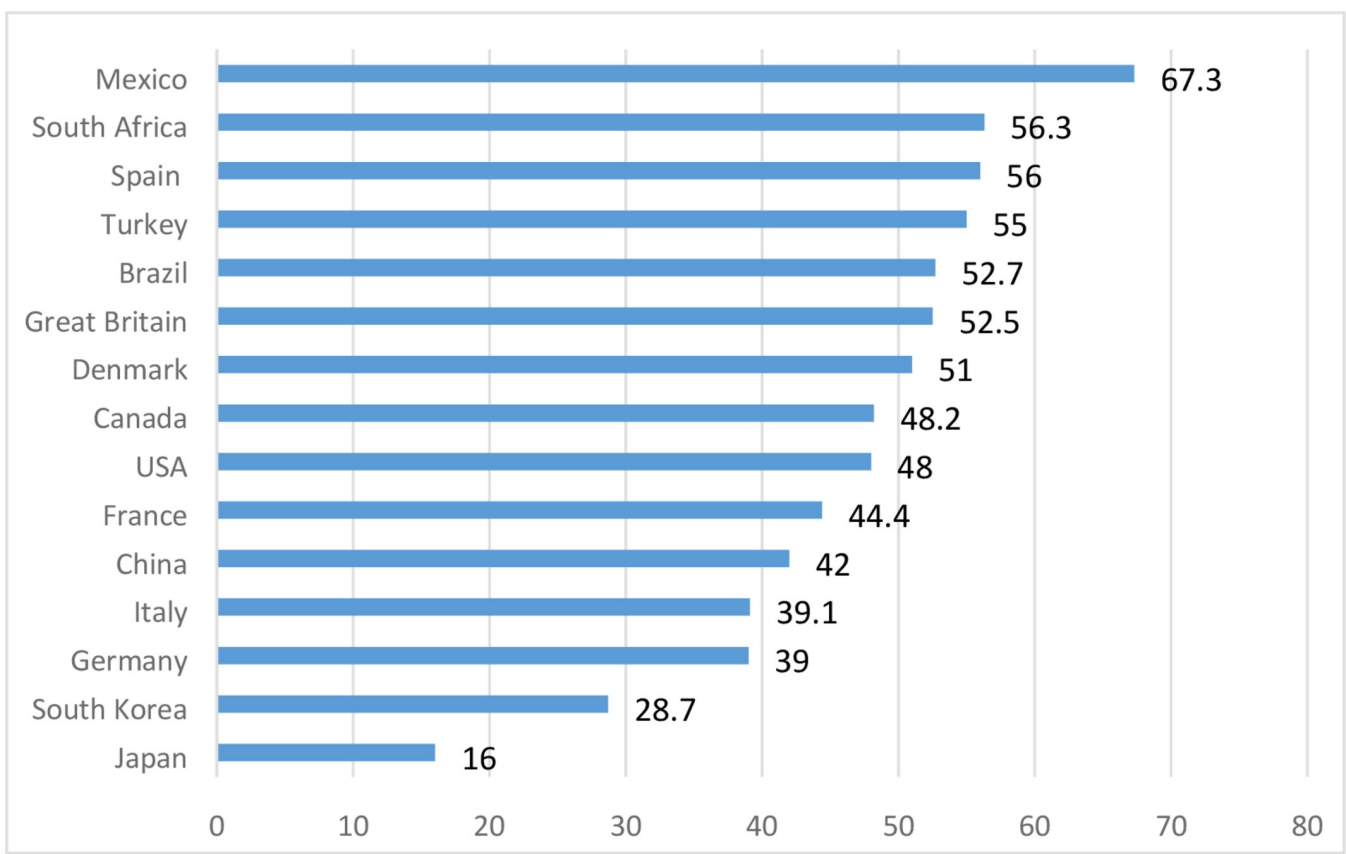

Figure 1 Country differences in manager responses in relation to depression: manager offered help to employee with depression.

seem to represent important societal indicators in their own right and probably influence openness about depression and performance in the workplace independently.

Interestingly, the positive active strategy by the manager to support the employee was associated with greater presenteeism, but not significantly associated with absenteeism. On the other hand, the negative reaction by a manager to avoid an employee with depression was particularly important in terms of greater absenteeism, but was not significantly related to presenteeism. It may be that a supportive manager is most important in helping employees to remain motivated and feel valued while performing their duties in the workplace. It could also be that if employees feel supported by their manager, then they will also feel that it is acceptable to take the time off to recover sufficiently so that when they return to work they have greater capacity to perform their workplace role. Working in a setting, where managers actively avoid employees with depression on the other hand, may encourage avoidant behaviour among those employees, resulting in a tendency to take more days off work.

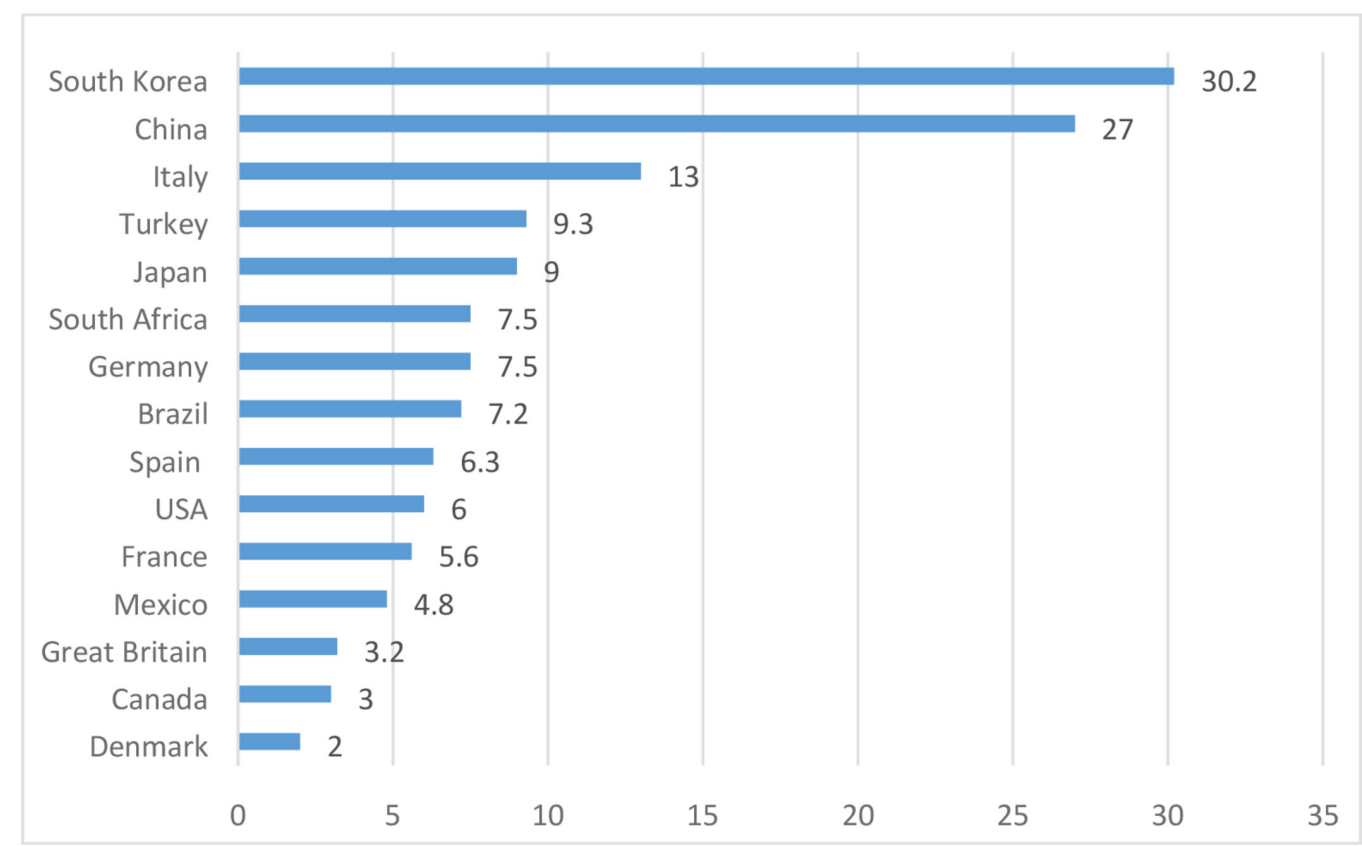

Figure 2 Country differences in manager responses in relation to depression: manager avoided talking about depression with the employee. 
Table 2 Individual, manager and country contextual characteristics associated with greater absenteeism among individuals with depression* (multivariable linear regression, $n=2715) \dagger$

Unadjusted GEE

parameter estimates

$(95 \% \mathrm{Cl})$

P values

Adjusted GEE parameter

estimates $(95 \% \mathrm{Cl}) \quad \mathrm{P}$ values

\section{Individual characteristics}

Gender

\begin{tabular}{|c|c|c|c|c|}
\hline Male & $0.47(-1.96$ to 2.89$)$ & 0.71 & $0.87(-0.49$ to 2.23$)$ & 0.21 \\
\hline Female & Reference & & Reference & \\
\hline \multicolumn{5}{|l|}{ Age } \\
\hline $45-64$ & $-3.64(-5.93$ to -1.35$)$ & 0.002 & $-1.16(-3.29$ to 0.96$)$ & 0.28 \\
\hline $25-44$ & $-8.70(-14.09$ to -3.31$)$ & 0.002 & $-3.88(-8.58$ to 0.81$)$ & 0.11 \\
\hline $16-24$ & Reference & & Reference & \\
\hline \multicolumn{5}{|l|}{ Education } \\
\hline High & 3.43 (2.05 to 4.83$)$ & $<0.0001$ & 2.29 (0.97 to 3.60$)$ & 0.0007 \\
\hline Medium & 2.56 (1.30 to 3.82$)$ & $<0.0001$ & $1.09(-1.01$ to 3.19$)$ & 0.31 \\
\hline Low & Reference & & Reference & \\
\hline Working in a larger company & $-0.93(-1.41$ to -0.45$)$ & $<0.0001$ & $-0.82(-1.54$ to -0.09$)$ & 0.03 \\
\hline \multicolumn{5}{|l|}{ Country contextual characteristics } \\
\hline Offered help & $-1.28(-2.92$ to 0.13$)$ & 0.05 & $-1.06(-2.39$ to 0.27$)$ & 0.12 \\
\hline Avoided talking about it & 7.27 (1.06 to 13.92$)$ & $<0.0001$ & 4.13 (1.68 to 6.57$)$ & 0.0009 \\
\hline Country GDP $\ddagger$ & 2.59 (1.87 to 3.30$)$ & $<0.0001$ & $2.97(-0.48$ to 6.42$)$ & 0.09 \\
\hline
\end{tabular}

*Unemployment rates were taken from the International Labour Organization http://www.ilo.org/global/research/global-reports/globalemployment-trends/2014/WCMS_233936/lang-en/index.htm.

†Model controlled for country dummy variables.

‡GDP taken from the World Bank: http://data.worldbank.org/indicator/NY.GDP.PCAP.CD.

GEE, generalised estimating equation.

Our previous work found that working in a context where managers are actively supporting employees with depression was associated with greater comfort around the issue of depression among employees ${ }^{3}$ and more openness among those with depression. Additionally, managers who have support and training in dealing with mental health issues are more likely to recognise and act on problems earlier, which can prevent further worsening of the problem. ${ }^{31}$ However, some literature has noted a tendency among management to instigate disciplinary sanctions as a way to improve productivity among underperforming employees rather than trying to understand the underlying issues and provide support to overcome them. ${ }^{32}$ This may increase concealment of problems and thereby work against facilitating an environment of social acceptance and disclosure ${ }^{33}$ which could be important to optimising productivity.

There were also some individual-level factors associated with absenteeism and presenteeism. In relation to presenteeism, our findings suggest that female individuals with low education and those in the middle age group (25-44) might need more support in the workplace as they tended to report lower levels of presenteeism in relation to their depression. Interestingly, these characteristics are also common risk factors associated with depression ${ }^{34-36}$ and it may be that these individuals are more impacted on by depressive symptoms and/or have greater levels of severity.

In relation to absenteeism, individuals working for small companies and with high levels of education tended to take more days off work. Although it is well established that education and higher socioeconomic group more generally are inversely related with prevalence of depression, the link between education and absenteeism due to depression seems less clear from the literature. One systematic review found only limited evidence to support a relationship between increased work disability and low education. ${ }^{37}$ However, when looking at absenteeism in particular, other large studies, based on nationally representative populations have found that absenteeism associated with depression was higher among those with more education and higher incomes. ${ }^{38}$ It may be that productivity losses are higher among employees with jobs which require skilled decision-making and communication which may be associated with higher education levels. Those with higher education levels and higher pay have more control over their jobs and working hours compared with those with lower education and lower salaries whose jobs are often more vulnerable and less flexible. Other research has also shown that individuals working in smaller companies ${ }^{79}$ tend to have higher 
Table 3 Individual, manager and country contextual characteristics associated with greater presenteeism among individuals with depression* (multivariable linear regression, $n=2715) \dagger$

Unadjusted GEE parameter estimates $(95 \% \mathrm{Cl})$

P values

Adjusted GEE parameter estimates $(95 \% \mathrm{Cl}) \quad P$ values

Individual characteristics

Gender

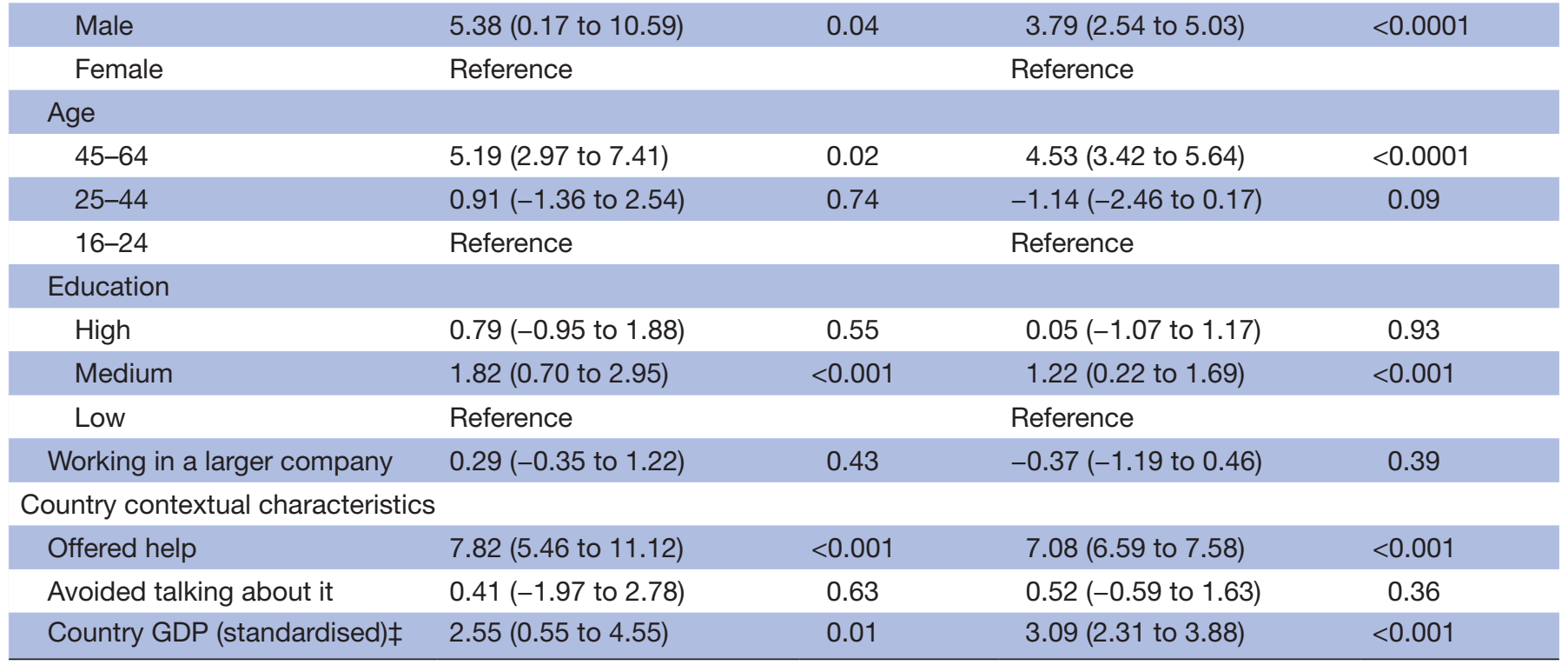

*Unemployment rates were taken from the International Labour Organization http://www.ilo.org/global/research/global-reports/globalemployment-trends/2014/WCMS_233936/lang-en/index.htm.

†Model controlled for country dummy variables.

‡GDP taken from the World Bank: http://data.worldbank.org/indicator/NY.GDP.PCAP.CD.

GEE, generalised estimating equation.

levels of absenteeism. It may be that large companies offer more structure in terms of transitioning back to work, including offering part-time return to work. It has also been suggested that smaller companies have lower awareness of the resources available to them to support employees with mental health problems. ${ }^{20}$

\section{Strengths and limitations}

Our study addresses a gap in the literature in terms of developing our understanding of how manager reactions and workplace practices are associated with productivity among individuals with depression. Our findings come from a unique dataset including both employees and managers from 15 diverse countries, and information on employees' and mangers' experiences of depression in the workplace. The sample was designed to be geographically representative of each country.

There are, however, several limitations which need to be considered. Although symptom severity is an important factor related to workplace productivity, ${ }^{40}$ unfortunately the survey was not able to collect information on severity or type of symptoms. Additionally, depression diagnosis was determined via self-report. Nevertheless, the distribution of characteristics among respondents with depression are similar to other epidemiological studies, as study respondents reporting a diagnosis of depression were more likely to be female, divorced and working part time. In addition, prevalence of depression diagnosis was lowest in Asian countries. ${ }^{34} 35$ and Italy had the lowest prevalence within the European countries. ${ }^{41}$ Additionally, the anonymised format of data collection online increased participants' willingness to disclose mental health problems. ${ }^{42}$ Although we feel that these data provide an initial important step to understand depression in the workplace in relation to managers reactions and productivity across diverse settings, the results should be interpreted with caution given these limitations.

We recognise that national mental health policies, employment assistance programmes available in the workplace and other policies could be important factors which help explain relationships between depression and productivity in the workplace, and it is a limitation that we were not able to include this information in our analyses. There is considerable within-country variation in terms of support and understanding for depression, but this paper focuses on the broad macro-level factors. Given the relationships which we identified by averaging reactions and practices within countries, our findings suggest that broad country-level policies can make a difference to the lives of individuals with depression. Additional limitations are that data from this study did not include information 
on variables such as ethnicity or migration which might be associated with workplace exclusion, as well as associations with mental illness. Additionally, response rates were relatively low. Finally, these data were cross-sectional, so it was not possible to examine pathways or mechanisms to increased productivity.

\section{CONCLUSIONS}

This study highlights the importance of effective policies and practices which help managers to actively support employees with depression, including strategies to facilitate better workplace performance. The business case for intervention through better managerial response is exemplified by the substantial costs associated with mental health problems and evidence from a number of studies that mental health can improve through workplace programmes, with economic benefits to employers. ${ }^{434}$ Consequently, benefits which would result from a well-implemented support programme should encourage employers to act. Support is needed for managers to directly support employees to feel open and comfortable in discussing mental health issues. This is in addition to programmes which effectively facilitate early intervention practices and support for and recognition of depression among employees, as well as clear transition and referral pathways for employees who need to take time off and for those returning to work.

Acknowledgements The authors would like to acknowledge $\mathrm{H}$ Lundbeck $\mathrm{A} / \mathrm{S}$ and IPSOS Mori for sharing the IDEA survey data.

Contributors The original study design and protocol were written by SE-L and MK. SE-L performed data analysis and initial drafting of the manuscript with contributions from MK. Both authors participated in the interpretation of the analysis, editing and rewriting of the manuscript and approved the final manuscript.

Funding Funding for this study was provided by $\mathrm{H}$ Lundbeck A/S. H Lundbeck A/S put together the questionnaire with the European Depression Association.

Competing interests SE-L and MK received consulting fees from $H$ Lundbeck A/S.

Patient consent Not required.

Provenance and peer review Not commissioned; externally peer reviewed.

Data sharing statement № additional unpublished data are available.

Open access This is an open access article distributed in accordance with the Creative Commons Attribution Non Commercial (CC BY-NC 4.0) license, which permits others to distribute, remix, adapt, build upon this work non-commercially, and license their derivative works on different terms, provided the original work is properly cited and the use is non-commercial. See: http://creativecommons.org/ licenses/by-nc/4.0/

(C) Article author(s) (or their employer(s) unless otherwise stated in the text of the article) 2018. All rights reserved. No commercial use is permitted unless otherwise expressly granted.

\section{REFERENCES}

1. Corker E, Hamilton S, Robinson E, et al. Viewpoint survey of mental health service users' experiences of discrimination in England 20082014. Acta Psychiatr Scand 2016;134:6-13.

2. Lasalvia A, Zoppei S, Van Bortel T, et al. ASPEN/INDIGO Study Group. Global pattern of experienced and anticipated discrimination reported by people with major depressive disorder: a cross-sectional survey. Lancet 2013;381:55-62.
3. Evans-Lacko S, Knapp M. Importance of social and cultural factors for attitudes, disclosure and time off work for depression: findings from a seven country European study on depression in the workplace. PLoS One 2014;9:e91053.

4. OECD. Sick on the Job?: Myths and Realities about Mental Health and Work, Mental Health and Work. Paris: OECD Publishing, 2012.

5. Brohan E, Henderson C, Wheat K, et al. Systematic review of beliefs, behaviours and influencing factors associated with disclosure of a mental health problem in the workplace. BMC Psychiatry 2012;12:11.

6. Dewa CS. Worker attitudes towards mental health problems and disclosure. Int J Occup Environ Med 2014;5:175-86.

7. Biggs D, Hovey N, Tyson PJ, et al. Employer and employment agency attitudes towards employing individuals with mental health needs. J Ment Health 2010;19:505-16.

8. Henderson C, Williams P, Little K, et al. Mental health problems in the workplace: changes in employers' knowledge, attitudes and practices in England 2006-2010. Br J Psychiatry Suppl 2013;55:s70-6.

9. Marmot M. Fair Society, Healthy Lives: Strategic review of health inequalities in England post-2010 (The Marmot Review). $2010 \mathrm{http}: / /$ www.ucl.ac.uk/gheg/marmotreview/.

10. Ekman M, Granström O, Omérov S, et al. The societal cost of depression: evidence from 10,000 Swedish patients in psychiatric care. J Affect Disord 2013;150:790-7.

11. Evans-Lacko S, Knapp M. Global patterns of workplace productivity for people with depression: absenteeism and presenteeism costs across eight diverse countries. Soc Psychiatry Psychiatr Epidemiol 2016;51:1525-37.

12. Cancelliere C, Cassidy JD, Ammendolia C, et al. Are workplace health promotion programs effective at improving presenteeism in workers? A systematic review and best evidence synthesis of the literature. BMC Public Health 2011:11:395.

13. World Bank. GDP per capita. Washington, DC: World Bank, 2014.

14. Kessler RC, Barber C, Beck A, et al. The World Health Organization Health and Work Performance Questionnaire (HPQ). J Occup Environ Med 2003;45:156-74.

15. Kessler RC, Ames M, Hymel PA, et al. Using the World Health Organization Health and Work Performance Questionnaire (HPQ) to evaluate the indirect workplace costs of illness. J Occup Environ Med 2004;46:S23-37.

16. Zeger SL, Liang KY. Longitudinal data analysis for discrete and continuous outcomes. Biometrics 1986;42:121-30.

17. Pan W. Akaike's information criterion in generalized estimating equations. Biometrics 2001;57:120-5.

18. Birnbaum HG, Kessler RC, Kelley D, et al. Employer burden of mild, moderate, and severe major depressive disorder: mental health services utilization and costs, and work performance. Depress Anxiety 2010;27:78-89.

19. Wagner SL, Koehn C, White Ml, et al. Mental Health Interventions in the Workplace and Work Outcomes: A Best-Evidence Synthesis of Systematic Reviews. Int J Occup Environ Med 2016;7:1-14.

20. McDaid D. Mental health in workplace settings, Luxembourg. 2008 http://ec.europa.eu/health/ph_determinants/life_style/mental/docs/ consensus_workplace_en.pdf.

21. Mind OF. Fit Job: From Evidence to Practice in Mental Health and Work, Mental Health and Work. Paris. 2015 http://www.oecd-ilibrary. org/employment/fit-mind-fit-job_9789264228283-en.

22. Bloom DE, Cafiero ET, Jané-Llopis E, et al. The Global Economic Burden of Noncommunicable Diseases, Geneva. 2011 http://www3. weforum.org/docs/WEF_Harvard_HE_GlobalEconomicBurdenNonC ommunicableDiseases_2011.pdf.

23. Evans-Lacko S, Knapp M, McCrone P, et al. The mental health consequences of the recession: economic hardship and employment of people with mental health problems in 27 European countries. PLoS One 2013;8:e69792.

24. Yang LH, Kleinman A. 'Face' and the embodiment of stigma in China: the cases of schizophrenia and AIDS. Soc Sci Med 2008;67:398-408.

25. Ando S, Yamaguchi S, Aoki Y, et al. Review of mental-health-related stigma in Japan. Psychiatry Clin Neurosci 2013;67:471-82.

26. Corrigan PW, Shapiro JR. Measuring the impact of programs that challenge the public stigma of mental illness. Clin Psychol Rev 2010;30:907-22.

27. Richards M, Hori H, Sartorius N, et al. Cross-cultural comparisons of attitudes toward schizophrenia amongst the general population and physicians: a series of web-based surveys in Japan and the United States. Psychiatry Res 2014;215:300-7.

28. Evans-Lacko S, Brohan E, Mojtabai R, et al. Association between public views of mental illness and self-stigma among individuals with mental illness in 14 European countries. Psychol Med 2012;42:1741-52. 
29. Lewer D, O'Reilly C, Mojtabai R, et al. Antidepressant use in 27 European countries: associations with sociodemographic, cultural and economic factors. Br J Psychiatry 2015;207:221-6.

30. Schomerus G, Evans-Lacko S, Rüsch N, et al. Collective levels of stigma and national suicide rates in 25 European countries. Epidemiol Psychiatr Sci 2015;24:1-6.

31. European Commission. Mental health compass database of policies and good practices. Masto project to reduce depression-related work disability. 2015 https://webgate.ec.europa.eu/sanco_mental health/public/POLICY/422/show.html (accessed 31 Jul 2015).

32. Lockwood G, Henderson C, Stansfeld S. An assessment of employer liability for workplace stress. Int J Law Manag 2017;59:202-16.

33. Brohan E, Evans-Lacko S, Henderson C, et al. Disclosure of a mental health problem in the employment context: qualitative study of beliefs and experiences. Epidemiol Psychiatr Sci 2014;23:289-300.

34. Kessler RC, Akiskal HS, Ames M, et al. Prevalence and effects of mood disorders on work performance in a nationally representative sample of U.S. workers. Am J Psychiatry 2006;163:1561-8.

35. Kessler RC, Bromet EJ. The epidemiology of depression across cultures. Annu Rev Public Health 2013;34:119-38.

36. Alonso J, Buron A, Rojas-Farreras S, et al. Perceived stigma among individuals with common mental disorders. J Affect Disord 2009;118:180-6.

37. Lagerveld SE, Blonk RW, Brenninkmeijer V, et al. Workfocused treatment of common mental disorders and return to work: a comparative outcome study. J Occup Health Psychol 2012;17:220-34.

38. Munce SE, Stansfeld SA, Blackmore ER, et al. The role of depression and chronic pain conditions in absenteeism: results from a national epidemiologic survey. J Occup Environ Med 2007;49:1206-11.

39. Koopmans PC, Roelen CA, Groothoff JW. Sickness absence due to depressive symptoms. Int Arch Occup Environ Health 2008;81:711-9.

40. Trivedi MH, Morris DW, Wisniewski SR, et al. Increase in work productivity of depressed individuals with improvement in depressive symptom severity. Am J Psychiatry 2013;170:633-41.

41. de Girolamo G, Polidori G, Morosini P, et al. Prevalence of common mental disorders in Italy. Soc Psychiatry Psychiatr Epidemiol 2006;41:853-61.

42. Henderson C, Evans-Lacko S, Flach C, et al. Responses to menta health stigma questions: the importance of social desirability and data collection method. Can J Psychiatry 2012;57:152-60.

43. Evans-Lacko S, Koeser L, Knapp M, et al. Evaluating the economic impact of screening and treatment for depression in the workplace. Eur Neuropsychopharmacol 2016;26:1004-13.

44. McDaid D, King D, Parsonage M. Workplace screening for depression and anxiety disorders. In: Knapp M, McDaid D, Parsonage M, eds. Mental health promotion and prevention: the economic case. London, UK: Personal Social Services Research Unit, London School of Economics and Political Science, 2011. 